\title{
Changes in leisure-time physical activity among Brazilian pregnant women: comparison between two birth cohort studies (2004 - 2015)
}

Carolina de Vargas Nunes Coll ${ }^{1 *}$, Marlos Rodrigues Domingues², Pedro Curi Hallal ${ }^{1,2}$, Inácio Crochemore Mohnsam da Silva ${ }^{1,2,3}$, Diego Garcia Bassani ${ }^{4,5}$, Alicia Matijasevich ${ }^{6}$, Aluísio Barros ${ }^{1}$, Iná S. Santos ${ }^{1}$ and Andréa Dâmaso Bertoldi ${ }^{1}$

\begin{abstract}
Background: Low levels of leisure-time physical activity (LTPA) during pregnancy have been shown in studies conducted worldwide. Surveillance is extremely important to monitor the progress of physical activity patterns over time and set goals for effective interventions to decrease inactivity among pregnant women. The aim of this study was to evaluate time changes in LTPA among Brazilian pregnant women in an 11-year period (2004-2015) by comparing data from two birth cohort studies.

Methods: Two population-based birth cohort studies were carried out in the city of Pelotas, southern Brazil, in 2004 and 2015. A total of 4244 and 4271 mothers were interviewed after delivery. Weekly frequency and duration of each session of LTPA in a typical week were reported for the pre-pregnancy period and for each trimester of pregnancy. Trends in both recommended LTPA ( $\geq 150 \mathrm{~min} /$ week) and any LTPA (regardless of weekly amount) were analysed overtime. Changes were also calculated separately for subgroups of maternal age, schooling, family income, parity, pre-pregnancy body mass index and pre-pregnancy LTPA.

Results: The proportion of women engaged in recommended levels of LTPA pre-pregnancy increased from 11.2\% (95\%Cl 10.0-12.2) in 2004 to $15.8 \%(95 \% \mathrm{Cl} 14.6-16.9)$ in 2015. During pregnancy, no changes were observed over the period for the first (10.6 to 10.9\%) and second (8.7 to 7.9\%) trimesters, whereas there was a decrease from 3.4\% $(95 \% \mathrm{Cl} 2.9-4.0)$ to $2.4 \%(95 \% \mathrm{Cl} 1.9-2.8)$ in the last trimester. Major decreases in LTPA in the last trimester were observed among women who were younger, with intermediate to high income, high schooling, primiparous, pre-pregnancy obese and, engaged in LTPA before pregnancy. Changes in any LTPA practice followed the same patterns described for recommended LTPA.
\end{abstract}

Conclusions: Despite the increase in the proportion of women engaged in LTPA before pregnancy between 2004-2015, LTPA levels remained stable during the first and second trimesters of pregnancy and declined during the third gestational trimester over the period. Interventions to encourage the maintenance of LTPA practice throughout pregnancy are urgently needed.

Keywords: Surveillance, Exercise, Physical activity, Motor activity, Pregnancy, Recommendations, Birth cohort studies

\footnotetext{
* Correspondence: carolinavncoll@gmail.com

${ }^{1}$ Postgraduate Program in Epidemiology, Federal University of Pelotas, Pelotas, Brazil

Full list of author information is available at the end of the article
} 


\section{Background}

Promotion of physical activity is a global public health priority due to it's potential to reduce the burden of noncommunicable diseases and improve the health of populations [1]. However, inactivity remains highly prevalent in most countries; nearly a quarter of the world's population does not meet the minimal physical activity recommendations [2]. In this scenario, adult women are more likely to be physically inactive compared to men and might be considered a priority group for planning strategies to increase physical activity levels [2]. Pregnancy is a period of particular concern since decreases in physical activity levels are observed during the childbearing years and may influence leisure-time physical activity (LTPA) patterns permanently over time $[3,4]$.

Low levels of LTPA during pregnancy are associated with complications that can influence maternal and child health such as gestational diabetes mellitus, excessive gestational weight gain, preeclampsia, depression, preterm birth, large for gestational age and increased neonatal adiposity [5-8]. Moreover, LTPA promotion could reduce the risk of long-term chronic diseases in the pregnant women [9] and the offspring [10].

Most of the current guidelines for physical activity during pregnancy recommend at least $150 \mathrm{~min}$ of moderate-intensity physical activity throughout the week, unless there is a medical reason to avoid physical activity $[11,12]$. Although LTPA during pregnancy offers minimal risks and has been shown to benefit most women [12], unacceptably low levels of LTPA during pregnancy have been shown in multiple studies worldwide [13-15]. In this context, population-based studies that allow monitoring the progress of LTPA patterns over time and set goals for effective interventions to increase physical activity levels during pregnancy are extremely important.

In spite of the increasingly efforts to consistently monitor global physical activity patterns and the substantial progress made in surveillance in recent years [2], data on physical activity patterns for subgroups at high risk of inactivity in the population, such as pregnant women, are still missing. To date, only one study describing changes in physical activity patterns among pregnant women over time has been found in the worldwide literature [16]. Findings of this surveillance study carried out in the United States of America (USA) over an 8-year period (1999-2006) revealed no change in the proportion of pregnant women meeting the minimal recommendations for physical activity (21.6 vs. $24.3 \%$ ). On the other hand, the proportion of women reporting any moderate LTPA practice increased from $46.8 \%$ in 1999-2002 to 58\% in 2003-06.

In Pelotas, southern Brazil, $12.9 \%$ of the mothers from the 2004 Pelotas Birth Cohort Study reported to engage in any LTPA during pregnancy and only $4.3 \%$ reported continued LTPA during the whole pregnancy, regardless of frequency and weekly amount [13]. While there is a growing body of worldwide literature and accessible information regarding the benefits of physical activity during pregnancy in the last decade [17], no specific public strategy that could have influenced LTPA levels among pregnant women was implemented in the city at the population level. The aim of the present study is to describe changes in LTPA among Brazilian pregnant women over an 11-year period by comparing data from two Birth Cohort Studies carried out in 2004 and 2015. Changes of LTPA patterns according to maternal age, education, parity, income, pre-pregnancy Body Mass Index (BMI) and pre-pregnancy LTPA are also reported.

\section{Methods}

\section{Research setting and study design}

The present study analyzed data from two populationbased birth cohort studies carried out in the city of Pelotas, Southern Brazil, in 2004 and 2015. Strategies to recruit participants were identical in both surveys. All five maternity hospitals (attending patients from both private and public insurance) located in Pelotas were visited daily from 1 January to 31 December of each year, and all births of mothers living in the urban areas of the city were identified (99\% of deliveries are performed at hospitals). A total of 4244 and 4271 mothers were interviewed after delivery in 2004 and 2015, respectively. The non-response rate at recruitment was below $1.5 \%$ in both studies. Face-to-face interviews took place in the hospital within $24 \mathrm{~h}$ after the delivery. Trained interviewers collected information on mother-child health using a structured questionnaire. Interviews lasted $60 \mathrm{~min}$, on average. A fieldwork supervisor repeated $10 \%$ of the interviews to check the quality of the information collected. Further methodological details of the 2004 study are available elsewhere $[13,18]$.

\section{Outcome measures and covariates}

Type, frequency and average duration of sessions of LTPA in a typical week (7-day recall) were investigated in four time periods during the perinatal interview: the three-month period prior to pregnancy as well as the first, second and third trimesters of pregnancy. Up to three different physical activities were recorded for each period. Women were asked not to report commuting, household or occupational activities as LTPA. The instrument used to assess LTPA in 2015 was the same employed in 2004 [13]. The total LTPA score was generated by the sum of minutes per week spent on each activity. A cut-off point of $150 \mathrm{~min}$ per week was used to classify women as active or not in each period. We also explored trends in any LTPA practice by comparing 
the proportion of women who were engaged in LTPA regardless of weekly amount.

Maternal covariates assessed in the studies and used in the comparison analysis were maternal age, schooling, parity, household income, pre-pregnancy BMI, and prepregnancy LTPA. Maternal age was collected as a continuous variable and divided into four categories (13-19, 20-29, 30-39 and 40-47 years). Schooling was assessed as maternal years of formal education and categorized into four categories $(0-4,5-8,9-11,12$ or more years). Family monthly income was assessed as the sum of incomes of household members in the past month and categorized into quintiles. Parity was categorized into three categories $(1 / 2 / \geq 3)$ according to the total number of live births, including the birth from the cohort. Pre-pregnancy BMI was categorized according to the World Health Organization criteria into underweight $\left(<18.5 \mathrm{~kg} / \mathrm{m}^{2}\right)$, normal weight $\left(18.5-24.9 \mathrm{~kg} / \mathrm{m}^{2}\right)$; overweigh $\left(25.0-29.9 \mathrm{~kg} / \mathrm{m}^{2}\right)$ and obese $\left(\geq 30 \mathrm{~kg} / \mathrm{m}^{2}\right)$ based on self-reported height and weight. Pre-pregnancy LTPA ( $\geq 150 \mathrm{~min} /$ week) was also considered as a covariate. Independent variables were identically collected in both surveys for comparability.

\section{Statistical analysis}

Statistical analyses were carried out using Stata version 13.0 (StataCorp, College Station, TX, USA). Data analysis initially included the comparison of study populations in terms of sociodemographic, behavioral and health characteristics. Percentages and confidence intervals were used to compare the data between the studies. The prevalence of LTPA was estimated for each study, and its changes in the period were evaluated, including comparisons according to subgroups of the independent variables. The prevalence of each type of LTPA practiced was also compared between the studies. Chi-square test for difference in proportions across the study period was used. To identify correlates of LTPA in each period, the proportion of active women was described according to the subgroups of the independent variables and confidence intervals were calculated. In each year, logistic regression models were used to provide estimates for the adjusted odds ratios within each subgroup (with adjustment for the other variables studied). Associations between LTPA during pregnancy (outcome) and maternal age, income, schooling and parity were not adjusted for pre-pregnancy variables (BMI and LTPA) because they were considered mediators in the causal chain of its determination. Statistical significance was set at $p<0.05$. We excluded from our analysis a group of 142 pregnant women from the 2015 Pelotas (Brazil) Birth Cohort Study who were randomly enrolled in the intervention group of a randomized controlled trial nested in the cohort to study the effects of an exercise program during pregnancy on mother and child health outcomes [19].

\section{Results}

\section{Samples description}

Data from a total of 4244 mothers in 2004 and 4129 mothers in 2015 were analysed in this study. Table 1 presents the mothers' sociodemographic, behavior and health-related characteristics in both cohorts. The proportion of adolescent pregnancies decreased from 19.0\% (95\%CI $17.9-20.2)$ in 2004 to $15.0 \%(14.0-16.1)$ in 2015 , while the proportion of mothers aged 30 to 39 years increased from $28.0 \%$ (95\%CI $26.7-29.3)$ to $34.7 \%$ (95\%CI 33.3-36.2). We observed an increase in the proportion of women with 12 or more years of formal education (9.9 to 30.0\%). Employment during pregnancy increased from $40.1 \%(95 \%$ CI $38.6-41.5)$ to $55.5 \%$ (95\%CI $58.5-$ 61.4) during the period. A decrease in the proportion of women who reported being underweight before pregnancy from $7.4 \%(95 \% \mathrm{CI} 6.5-8.4)$ in 2004 to $3.9 \%$ (95\%CI 3.3-4.5) in 2015, accompanied by an increase in the proportion of overweight (20.1 to $27.4 \%$ ) and obese (8.9 to $20.6 \%$ ) was observed. Smoking during pregnancy significantly decreased between 2004 and 2015 (27.6 to $17.0 \%$ ) while gestational diabetes nearly tripled (3.0 to $8.6 \%)$. Physical activity advice received from health professionals during prenatal care significantly decreased from $72.2 \%(95 \%$ CI $70.8-73.5)$ to $61.3 \%(95 \%$ CI $59.8-$ 62.8) from 2004 to 2015 . Over the period there was an increase in the median of family income from \$206 in 2004 to $\$ 649$ in 2015 (data not shown).

\section{Prevalence and changes in LTPA during pregnancy}

Changes in LTPA patterns are presented in Fig. 1. There was an increase in the proportion of women practicing any pre-pregnancy LTPA between 2004 and 2015, from $15.3 \%$ (95\%CI $14.2-16.3$ ) to $21.3 \%$ (95\%CI $20.1-22.6$ ); as well as in the proportion of those engaged in recommended levels of LTPA, from 11.2\% (95\%CI 10.0-12.2) to $15.8 \%$ (95\%CI 14.6-16.9). Regarding pregnancy LTPA patterns, no changes were observed for the first and second trimesters in the 11-year period. However, we observed a significant decrease in the prevalence of any LTPA from $6.6 \%(95 \%$ CI $5.9-7.5)$ to $3.4 \%$ (95\%CI $2.9-$ 4.0) as well as in recommended LTPA during the third trimester of pregnancy, declining from 5.0\% (95\% CI 4.35.7 ) to $2.4 \%$ (95\%CI $1.9-2.8$ ). In both cohort studies, the prevalence of LTPA (any or recommended) markedly declined from pre-pregnancy to the third gestational trimester $(p<0.001)$.

\section{Changes in patterns of LTPA practice during pregnancy}

Among women engaged in LTPA during pregnancy, walking was the most reported type of LTPA in both surveys (Fig. 2). The second most commonly reported LTPA was cycling and weight training in 2004 and 2015, respectively. Regarding changes over time, reductions in 
Table 1 Characteristics of the mothers in 2004 and 2015 Birth Cohort Studies. Pelotas, Brazil

\begin{tabular}{|c|c|c|c|c|}
\hline \multirow[t]{2}{*}{ Variables } & \multicolumn{2}{|c|}{$\begin{array}{l}2004 \\
(n=4244)\end{array}$} & \multicolumn{2}{|c|}{$\begin{array}{l}2015 \\
(n=4129)\end{array}$} \\
\hline & N & $\%(95 \% \mathrm{Cl})$ & N & $\%(95 \% \mathrm{Cl})$ \\
\hline Age (years) & $n=4242$ & & $n=4128$ & \\
\hline $12-19$ & 848 & $19.0(17.9-20.2)$ & 620 & $15.0(14.0-16.1)$ \\
\hline $20-29$ & 2224 & $49.8(48.2-51.2)$ & 1950 & $47.3(45.7-48.8)$ \\
\hline $30-39$ & 1251 & $28.0(26.7-29.3)$ & 1434 & $34.7(33.3-36.2)$ \\
\hline $40-47$ & 146 & $3.2(2.8-3.9)$ & 124 & $3.0(2.5-3.6)$ \\
\hline Skin Color & $n=4192$ & & $n=4122$ & \\
\hline White & 2581 & $61.6(60.1-63.0)$ & 2895 & $70.2(68.8-71.6)$ \\
\hline Non-white & 1611 & $38.4(37.0-39.9)$ & 1227 & $29.8(28.4-31.2)$ \\
\hline Schooling (years) & $n=4202$ & & $n=4127$ & \\
\hline $0-4$ & 658 & $15.7(14.6-16.8)$ & 390 & $9.5(8.6-10.4)$ \\
\hline $5-8$ & 1740 & $41.4(39.9-42.9)$ & 1082 & $26.2(24.9-27.6)$ \\
\hline $9-11$ & 1385 & $33.0(31.5-34.4)$ & 1415 & $34.3(32.9-35.7)$ \\
\hline$>=12$ & 419 & $9.9(9.1-10.9)$ & 1240 & $30.0(28.7-31.5)$ \\
\hline Marital status & $n=4244$ & & $n=4128$ & \\
\hline Living with a partner & 3542 & $83.5(82.3-84.5)$ & 3524 & $85.4(84.3-86.4)$ \\
\hline Living without a partner & 702 & $16.5(15.5-17.7)$ & 604 & $14.6(13.6-15.7)$ \\
\hline Employement during pregnancy & $n=4243$ & & $n=4128$ & \\
\hline Yes & 1700 & $40.1(38.6-41.5)$ & 2270 & $55.0(53.5-56.5)$ \\
\hline No & 2543 & $59.9(58.5-61.4)$ & 1858 & $45.0(43.5-46.5)$ \\
\hline Pre-pregnancy BMI (Kg/m²) & $n=2887$ & & $n=4005$ & \\
\hline$<18.5$ & 213 & $7.4(6.5-8.4)$ & 155 & $3.9(3.3-4.5)$ \\
\hline $18.5-24.9$ & 1836 & $63.6(61.8-65.3)$ & 1928 & $48.1(46.6-49.7)$ \\
\hline $25.0-29.9$ & 581 & $20.1(18.7-21.6)$ & 1096 & $27.4(26.0-28.8)$ \\
\hline$>=30$ & 257 & $8.9(7.9-10.0)$ & 826 & $20.6(19.4-21.9)$ \\
\hline Parity & $n=4243$ & & $n=4127$ & \\
\hline 1 (primiparae) & 1673 & $39.4(38.0-40.9)$ & 2047 & $49.6(48.1-51.1)$ \\
\hline 2 & 1105 & $26.1(24.7-27.4)$ & 1274 & $30.9(29.5-32.3)$ \\
\hline 3 or more & 1465 & $34.5(33.1-36.0)$ & 806 & $19.5(18.3-20.8)$ \\
\hline Smoking during pregnancy ${ }^{a}$ & $n=4244$ & & $n=4126$ & \\
\hline Yes & 1172 & $27.6(26.3-29.0)$ & 701 & $17.0(15.9-18.2)$ \\
\hline No & 3072 & $72.4(71.0-73.7)$ & 3425 & $83.0(81.8-84.1)$ \\
\hline Gestational diabetes mellitus ${ }^{b}$ & $n=4241$ & & $n=4125$ & \\
\hline Yes & 126 & $3.0(2.5-3.5)$ & 353 & $8.6(7.7-9.5)$ \\
\hline No & 4115 & $97.0(96.5-97.5)$ & 3772 & $91.4(90.5-92.3)$ \\
\hline Gestational hypertension ${ }^{\mathrm{b}}$ & $n=4236$ & & $n=4126$ & \\
\hline Yes & 1006 & $23.7(22.5-25.1)$ & 1055 & $25.6(24.3-26.9)$ \\
\hline No & 3230 & $76.3(74.9-77.5)$ & 3071 & $74.4(73.1-75.7)$ \\
\hline Physical activity counselling during prenatal care & $n=4155$ & & $n=4029$ & \\
\hline Yes & 2999 & $72.2(70.8-73.5)$ & 2471 & $61.3(59.8-62.8)$ \\
\hline No & 1156 & $27.8(26.5-29.2)$ & 1558 & $38.7(37.2-40.2)$ \\
\hline
\end{tabular}

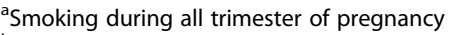

${ }^{b}$ Self-reported gestational diabetes mellitus and hypertension 


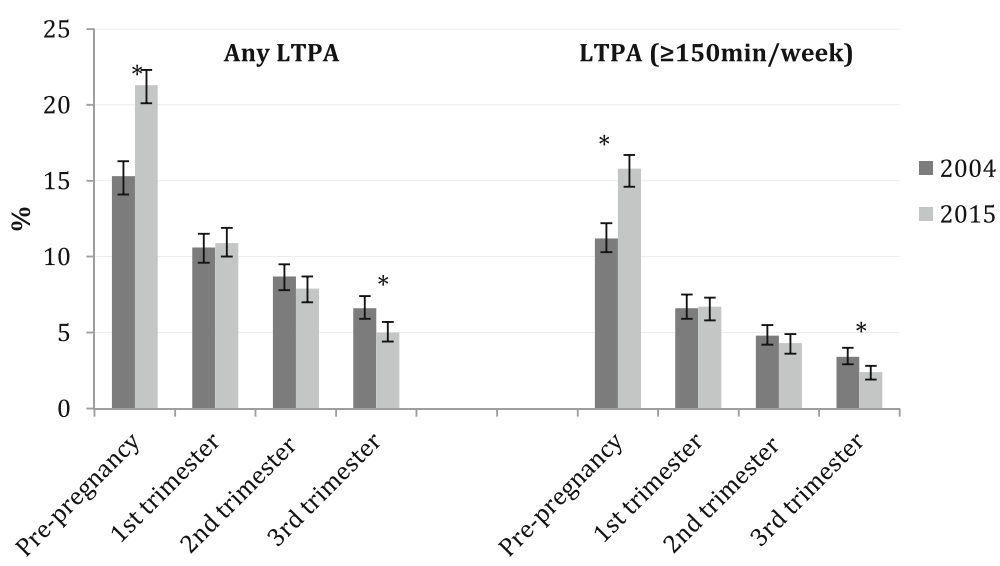

Fig. 1 Changes in Leisure-time Physical Activity patterns before and during pregnancy. Pelotas, Brazil, 2004-2015. * Significant changes

walking (from $77.2 \%$ in 2004 to $47.4 \%$ in 2015 ) and cycling (from $8 \%$ in 2004 to $3.1 \%$ in 2015 ) and increases in weight training (from $6.1 \%$ in 2004 to $21 \%$ in 2015), water gymnastics (from 3.9\% in 2004 to $9.7 \%$ in 2015), aerobics (from $2.7 \%$ in 2004 to $5.7 \%$ in 2015 ) and dancing (from $2 \%$ in 2004 to $4.9 \%$ in 2015 ) were observed.

\section{Changes in LTPA according to the subgroups of the independent variables}

Tables 2 and 3 describe detailed time changes in the prevalence of recommended and any LTPA by maternal age, income, schooling, parity, pre-pregnancy BMI and pre-pregnancy LTPA from 2004 to 2015 . The proportion of women considered active increased in the prepregnancy period among all groups of maternal age, income (except for the richest) and parity, from 2004 to 2015. Marked increases were also observed among those mothers with 5 to 8 years of formal education and those classified as underweight before the pregnancy. During the first and second trimesters of pregnancy, despite the overall stability in prevalence, decreases in recommended LTPA were observed for some subgroups of women. During the first gestational trimester, LTPA declined between 2004 and 2015 among women with 9 to 11 years of schooling and among those considered active before pregnancy. Decreases in the prevalence of recommended levels of LTPA in the second trimester of pregnancy were observed among mothers aged 20 to 29 years, classified in the intermediate quintile of family income, with at least 9 years of education, who were giving birth to the first child, obese and, among those engaged in recommended levels of LTPA before pregnancy. Declining changes for the third gestational trimester followed the same patterns observed for the second trimester of pregnancy, except that LTPA also declined among mothers belonging to the fourth quintile

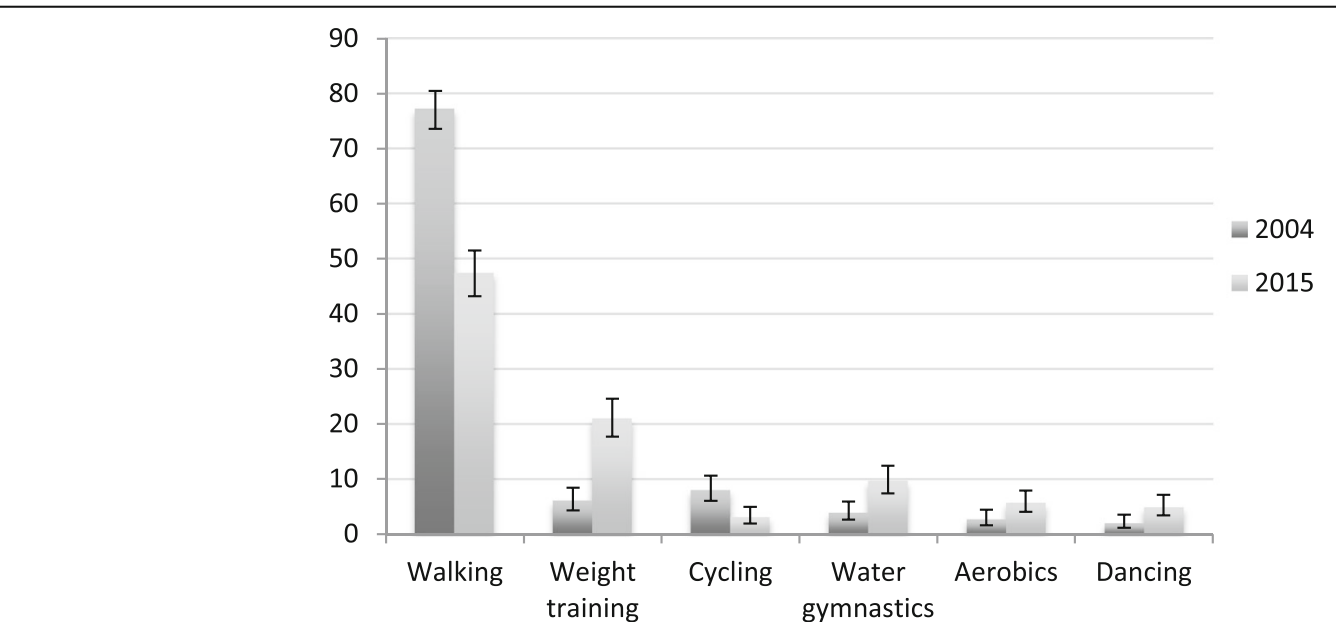

Fig. 2 Changes in type of Leisure-Time Physical Activity practiced during pregnancy. Pelotas, Brazil, 2004-2015. * Among those practicing any LTPA during pregnancy ( $N=562$ in 2004; $N=549$ in 2015). Only those activities with a significant change are presented 
Table 2 Changes in recommended LTPA ( $\geq 150$ min/week) by maternal age, schooling, income, parity, pre-pregnancy BMI and prepregnancy LTPA. Pelotas, Brazil, 2004-2015

\begin{tabular}{|c|c|c|c|c|c|c|c|c|c|c|c|c|}
\hline \multirow[b]{2}{*}{ Variables } & \multicolumn{2}{|c|}{$\begin{array}{l}\text { Pre-pregnancy } \\
N(\%)\end{array}$} & \multirow[t]{2}{*}{$\%$ change } & \multicolumn{2}{|c|}{$\begin{array}{l}\text { 1st trimester } \\
N(\%)\end{array}$} & \multirow[t]{2}{*}{$\%$ change } & \multicolumn{2}{|c|}{$\begin{array}{l}\text { 2nd trimester } \\
N(\%)\end{array}$} & \multirow[t]{2}{*}{$\%$ change } & \multicolumn{2}{|c|}{$\begin{array}{l}\text { 3rd trimester } \\
N(\%)\end{array}$} & \multirow[t]{2}{*}{$\%$ change } \\
\hline & 2004 & 2015 & & 2004 & 2015 & & 2004 & 2015 & & 2004 & 2015 & \\
\hline \multicolumn{13}{|c|}{ Maternal age (years) } \\
\hline $12-19$ & 7.8 & 12.3 & $+57.7^{* *}$ & 5.1 & 5.2 & +2.0 & 4.1 & 4.0 & -2.4 & 4.0 & 2.4 & -40.0 \\
\hline $20-29$ & 210.4 & 14.9 & $+43.3^{* *}$ & 6.3 & 5.3 & -15.9 & 5.0 & 3.1 & $-38.0^{* *}$ & 3.7 & 2.1 & $-43.2^{* *}$ \\
\hline $30-39$ & 15.4 & 18.3 & $+18.8^{*}$ & 8.4 & 9.0 & +7.1 & 5.6 & 5.9 & +5.4 & 3.1 & 2.8 & -9.7 \\
\hline $40-47$ & 8.5 & 16.9 & $+98.8^{*}$ & 4.3 & 8.9 & +107.0 & 1.4 & 4.8 & +242.9 & 1.4 & 2.4 & +71.4 \\
\hline \multicolumn{13}{|c|}{ Family income (quintiles) } \\
\hline 1 (poorest) & 5.3 & 11.7 & $+120.7^{* *}$ & 3.8 & 3.5 & -17.9 & 2.5 & 2.9 & +16.0 & 1.8 & 1.8 & 0 \\
\hline 2 & 6.0 & 11.6 & $+93.3^{* *}$ & 4.9 & 6.3 & +28.6 & 3.5 & 4.0 & +14.3 & 2.5 & 2.5 & 0 \\
\hline 3 & 9.1 & 15.3 & $+68.1^{* *}$ & 6.0 & 5.3 & -21.7 & 5.5 & 3.0 & $-35.5^{*}$ & 4.0 & 2.2 & $-35.0^{*}$ \\
\hline 4 & 12.7 & 16.7 & $+31.5^{*}$ & 6.8 & 7.8 & +14.7 & 4.7 & 3.4 & -27.7 & 3.4 & 1.5 & $-55.9^{*}$ \\
\hline 5 (wealthiest) & 23.7 & 26.8 & +13.1 & 11.7 & 11.8 & +0.9 & 8.2 & 9.2 & +12.2 & 5.6 & 4.7 & -16.1 \\
\hline \multicolumn{13}{|l|}{ Schooling (years) } \\
\hline $0-4$ & 4.3 & 5.6 & +30.2 & 2.9 & 3.3 & +13.8 & 2.0 & 2.6 & +30.0 & 1.2 & 2.1 & +75.0 \\
\hline $5-8$ & 7.8 & 10.6 & $+35.9^{*}$ & 4.6 & 4.5 & -12.2 & 3.3 & 3.0 & -9.1 & 2.7 & 2.0 & -25.9 \\
\hline $9-11$ & 13.4 & 14.3 & +6.7 & 8.2 & 5.2 & $-36.6^{* *}$ & 5.7 & 3.1 & $-45.6^{* *}$ & 3.8 & 2.0 & $-47.4^{* *}$ \\
\hline$\geq 12$ & 27.2 & 25.1 & -17.7 & 14.6 & 11.5 & -21.2 & 11.9 & 7.3 & $-18.0^{* *}$ & 7.6 & 3.2 & $-57.9^{* *}$ \\
\hline \multicolumn{13}{|l|}{ Parity } \\
\hline 1 & 13.9 & 18.7 & $+34.5^{* *}$ & 8.7 & 8.5 & -2.3 & 7.1 & 5.4 & $-23.9^{*}$ & 5.1 & 2.9 & $-43.2^{* *}$ \\
\hline 2 & 11.1 & 13.8 & $+24.3^{*}$ & 5.7 & 5.4 & -5.3 & 3.6 & 3.3 & -8.3 & 2.4 & 2.0 & -16.7 \\
\hline 3 or more & 8.3 & 11.4 & $+37.3^{*}$ & 4.8 & 4.2 & -12.5 & 3.1 & 2.9 & -6.5 & 2.2 & 1.6 & -27.3 \\
\hline \multicolumn{13}{|c|}{ Pre-pregnancy BMI } \\
\hline$<18.5$ & 5.6 & 11.6 & $+107.1^{*}$ & 4.2 & 5.8 & +38.1 & 3.8 & 3.2 & -15.8 & 1.4 & 3.2 & +128.6 \\
\hline $18.5-24.9$ & 14.0 & 16.1 & +15.0 & 7.8 & 7.2 & -17.7 & 6.1 & 5.2 & -14.8 & 4.6 & 2.8 & $-39.1^{* *}$ \\
\hline $25.0-29.9$ & 14.3 & 17.8 & +24.5 & 8.6 & 7.6 & -11.6 & 5.0 & 3.8 & -24.0 & 2.8 & 2.6 & -7.1 \\
\hline$>=30$ & 14.0 & 14.2 & +1.4 & 8.2 & 5.3 & -35.4 & 6.2 & 3.3 & $-46.8^{*}$ & 3.9 & 1.2 & $-69.2^{*}$ \\
\hline \multicolumn{13}{|c|}{ Pre-pregnancy LTPA ( $\geq 150 \mathrm{~min} /$ week) } \\
\hline Yes & & & & 47.7 & 34.8 & $-27.0^{* *}$ & 31.7 & 19.2 & $-39.4^{* *}$ & 19.3 & 9.7 & $-49.7^{* *}$ \\
\hline No & & & & 1.4 & 1.4 & 0 & 1.4 & 1.5 & +7.1 & 1.4 & 1.0 & -28.6 \\
\hline Total & 11.2 & 15.8 & $+41.1^{* *}$ & 6.6 & 6.7 & +1.5 & 4.8 & 4.3 & -10.4 & 3.4 & 2.4 & $-29.4^{* *}$ \\
\hline
\end{tabular}

${ }^{* *} X^{2}$ for change 2004-2015 $p<0.01$

${ }^{*} X^{2}$ for change 2004-2015 $p<0.05$

of family income and among those with a normal prepregnancy BMI.

The proportion of women engaged in any LTPA before pregnancy also increased from 2004 to 2015 and among all subgroups of maternal age, income, schooling, parity and, pre-pregnancy LTPA (Table 3). In terms of prepregnancy $\mathrm{BMI}$, marked increases in the prevalence of any LTPA were observed only among mothers classified as normal according to their pre-pregnancy BMI. During pregnancy the same patterns described for recommended LTPA were observed for any LTPA, except that in the third trimester of pregnancy it also decreased among adolescent mothers and those with intermediate schooling.

\section{Correlates of LTPA during pregnancy}

Adjusted associations between recommended LTPA and the independent variables in 2004 and 2015 are presented in Table 4. During the pre-pregnancy period, mothers aged 30 to 39 years were more likely to reach recommended LTPA levels when compared to adolescent mothers in 2004 (OR 1.69; 95\%CI 1.17-2.44) but no association between maternal age and pre-pregnancy LTPA was found in 2015. In both 2004 and 2015 studies, income 
Table 3 Changes in any LTPA by maternal age, schooling, income, parity, pre-pregnancy BMI and pre-pregnancy LTPA. Pelotas, Brazil, 2004-2015

\begin{tabular}{|c|c|c|c|c|c|c|c|c|c|c|c|c|}
\hline \multirow[b]{2}{*}{ Variables } & \multicolumn{2}{|c|}{$\begin{array}{l}\text { Pre-pregnancy } \\
N(\%)\end{array}$} & \multirow[t]{2}{*}{$\%$ change } & \multicolumn{2}{|c|}{$\begin{array}{l}\text { 1st trimester } \\
N(\%)\end{array}$} & \multirow[t]{2}{*}{$\%$ change } & \multicolumn{2}{|c|}{$\begin{array}{l}\text { 2nd trimester } \\
N(\%)\end{array}$} & \multirow[t]{2}{*}{ \%change } & \multicolumn{2}{|c|}{$\begin{array}{l}\text { 3rd trimester } \\
N(\%)\end{array}$} & \multirow[t]{2}{*}{$\%$ change } \\
\hline & 2004 & 2015 & & 2004 & 2015 & & 2004 & 2015 & & 2004 & 2015 & \\
\hline \multicolumn{13}{|c|}{ Maternal age (years) } \\
\hline $12-19$ & 11.3 & 16.6 & $+46.9^{* *}$ & 9.1 & 8.2 & -9.9 & 7.8 & 6.5 & -16.7 & 7.9 & 4.4 & $-44.3^{* *}$ \\
\hline $20-29$ & 14.2 & 19.2 & $+35.2^{* *}$ & 10.3 & 9.0 & -12.6 & 8.9 & 16.0 & $-32.6^{* *}$ & 6.8 & 4.4 & $-35.3^{* *}$ \\
\hline $30-39$ & 20.1 & 26.0 & $+29.4^{* *}$ & 12.4 & 14.5 & +16.9 & 9.6 & 11.1 & +15.6 & 5.9 & 6.3 & +6.8 \\
\hline $40-47$ & 12.8 & 23.4 & $+82.8^{*}$ & 7.8 & 14.5 & +85.9 & 4.3 & 7.3 & +69.8 & 2.8 & 3.3 & +17.9 \\
\hline \multicolumn{13}{|c|}{ Family income (quintiles) } \\
\hline 1 (poorest) & 7.3 & 14.9 & $+104.1^{* *}$ & 7.0 & 6.1 & -12.9 & 5.6 & 5.2 & -7.1 & 4.1 & 3.8 & -7.3 \\
\hline 2 & 9.5 & 15.5 & $+63.2^{* *}$ & 7.8 & 8.8 & +12.8 & 5.2 & 5.9 & +13.5 & 4.5 & 4.0 & -11.2 \\
\hline 3 & 12.6 & 18.3 & $+45.2^{* *}$ & 9.2 & 7.8 & -15.3 & 8.6 & 4.9 & $-43.0^{* *}$ & 7.1 & 3.6 & $-49.3^{* *}$ \\
\hline 4 & 17.1 & 22.9 & $+33.9^{* *}$ & 11.1 & 12.3 & +10.8 & 9.0 & 6.9 & -23.3 & 7.1 & 3.8 & $-46.8^{* *}$ \\
\hline 5 (wealthiest) & 30.2 & 40.2 & $+33.1^{* *}$ & 18.0 & 22.0 & +22.2 & 15.2 & 18.7 & +23.0 & 10.5 & 11.3 & +7.6 \\
\hline \multicolumn{13}{|l|}{ Schooling (years) } \\
\hline $0-4$ & 5.2 & 8.5 & $+63.5^{*}$ & 4.0 & 4.9 & +22.5 & 2.7 & 4.1 & +51.9 & 1.8 & 3.4 & +88.9 \\
\hline $5-8$ & 11.0 & 14.0 & $+27.3^{*}$ & 8.1 & 6.8 & -16.0 & 6.0 & 4.6 & -23.3 & 5.7 & 3.7 & $-35.1^{*}$ \\
\hline $9-11$ & 18.1 & 18.5 & +2.2 & 12.9 & 8.2 & $-36.4^{* *}$ & 10.5 & 5.0 & $-52.4^{* *}$ & 7.4 & 3.4 & $-54.1^{* *}$ \\
\hline$\geq 12$ & 36.8 & 35.0 & -4.9 & 22.7 & 19.6 & -13.7 & 21.5 & 15.1 & $-29.8^{* *}$ & 13.6 & 8.5 & $-62.5^{* *}$ \\
\hline \multicolumn{13}{|l|}{ Parity } \\
\hline 1 & 19.4 & 25.5 & $+31.4^{* *}$ & 14.1 & 13.8 & -2.1 & 13.0 & 10.1 & $-32.3^{* *}$ & 10.1 & 6.3 & $-37.6^{* *}$ \\
\hline 2 & 14.3 & 19.3 & $+35.0^{* *}$ & 9.7 & 9.7 & 0 & 7.0 & 6.5 & -7.1 & 5.3 & 4.2 & -20.8 \\
\hline 3 or more & 11.2 & 13.9 & +24.1 & 7.2 & 5.7 & -20.8 & 5.1 & 4.4 & -13.7 & 3.6 & 2.9 & -19.5 \\
\hline \multicolumn{13}{|c|}{ Pre-pregnancy BMI } \\
\hline$<18.5$ & 9.4 & 15.5 & +64.9 & 8.9 & 11.0 & +23.6 & 6.6 & 7.1 & +7.6 & 4.7 & 7.1 & +51.1 \\
\hline $18.5-24.9$ & 18.5 & 23.1 & $+24.9^{* *}$ & 12.6 & 12.6 & 0 & 10.9 & 9.6 & -11.9 & 8.7 & 6.9 & $-20.7^{*}$ \\
\hline $25.0-29.9$ & 18.9 & 22.1 & +16.9 & 13.1 & 11.0 & -16.0 & 8.6 & 7.0 & -18.6 & 5.6 & 4.1 & -26.8 \\
\hline$>=30$ & 8.7 & 18.8 & +0.5 & 11.7 & 8.2 & -29.9 & 10.1 & 5.9 & $-41.6^{*}$ & 5.8 & 2.1 & $-63.8^{* *}$ \\
\hline \multicolumn{13}{|c|}{ Pre-pregnancy LTPA (150 min/week) } \\
\hline Yes & & & & 55.9 & 40.4 & $-27.7^{* *}$ & 41.2 & 24.9 & $-39.6^{* *}$ & 27.1 & 14.5 & $-46.5^{* *}$ \\
\hline No & & & & 4.8 & 5.4 & +12.5 & 4.6 & 4.7 & +2.2 & 4.0 & 3.2 & -20.0 \\
\hline Total & 5.3 & 21.3 & $+39.2^{* *}$ & 10.6 & 10.9 & +2.8 & 8.7 & 7.9 & -9.2 & 6.6 & 5.0 & $-16.7^{* *}$ \\
\hline
\end{tabular}

$* * X^{2}$ for change 2004-2015 $p<0.01$

$* X^{2}$ for change 2004-2015 $p<0.05$

and schooling were positively associated with prepregnancy recommended LTPA while parity was negatively associated. Pre-pregnancy LTPA was the strongest correlate of LTPA during pregnancy in all gestational trimesters and in both surveys. However, the magnitude of associations was smaller in 2015. During pregnancy, maternal age was associated with LTPA in the first trimester of pregnancy in 2004; with mothers aged 30 to 39 years being more likely to be engaged in recommended LTPA. In 2015, all confidence intervals included the null value. A significant positive association between recommended LTPA and income was identified only for the first trimester in the 2004 cohort. A positive association between recommended LTPA and schooling was observed in all trimesters in 2004, but in 2015 it was only observed for the first trimester. Parity was negatively associated with LTPA in all trimesters of pregnancy in both studies, except in the third trimester in 2015.

In terms of any LTPA, similar patterns of associations were observed for all independent variables (Table 5). However, positive associations with income were observed in all pregnancy trimesters in 2015 and negative associations with parity were observed in all periods for both studies. Besides, negative associations between prepregnancy BMI and any LTPA were observed for all pregnancy trimesters in 2015. 


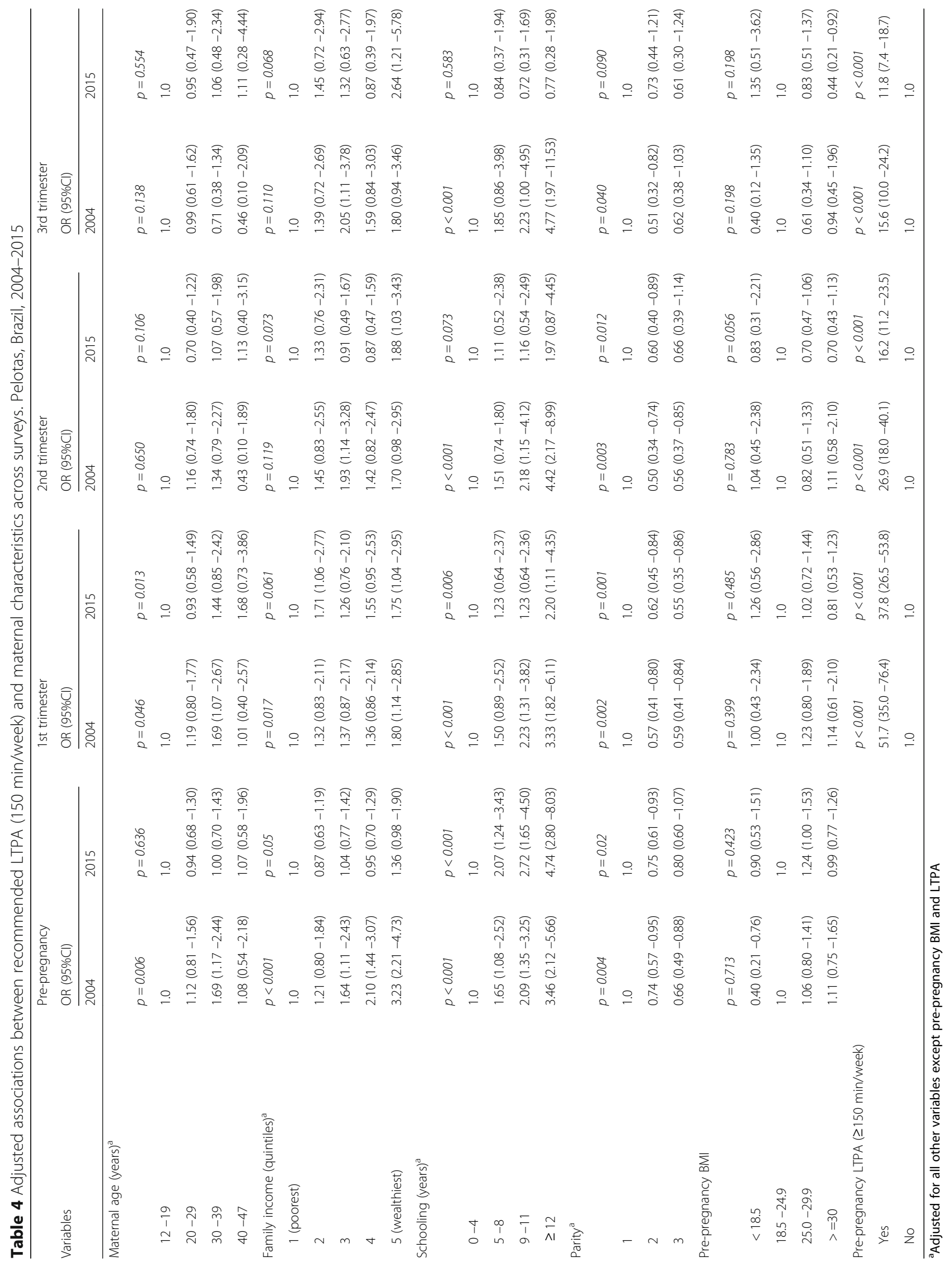




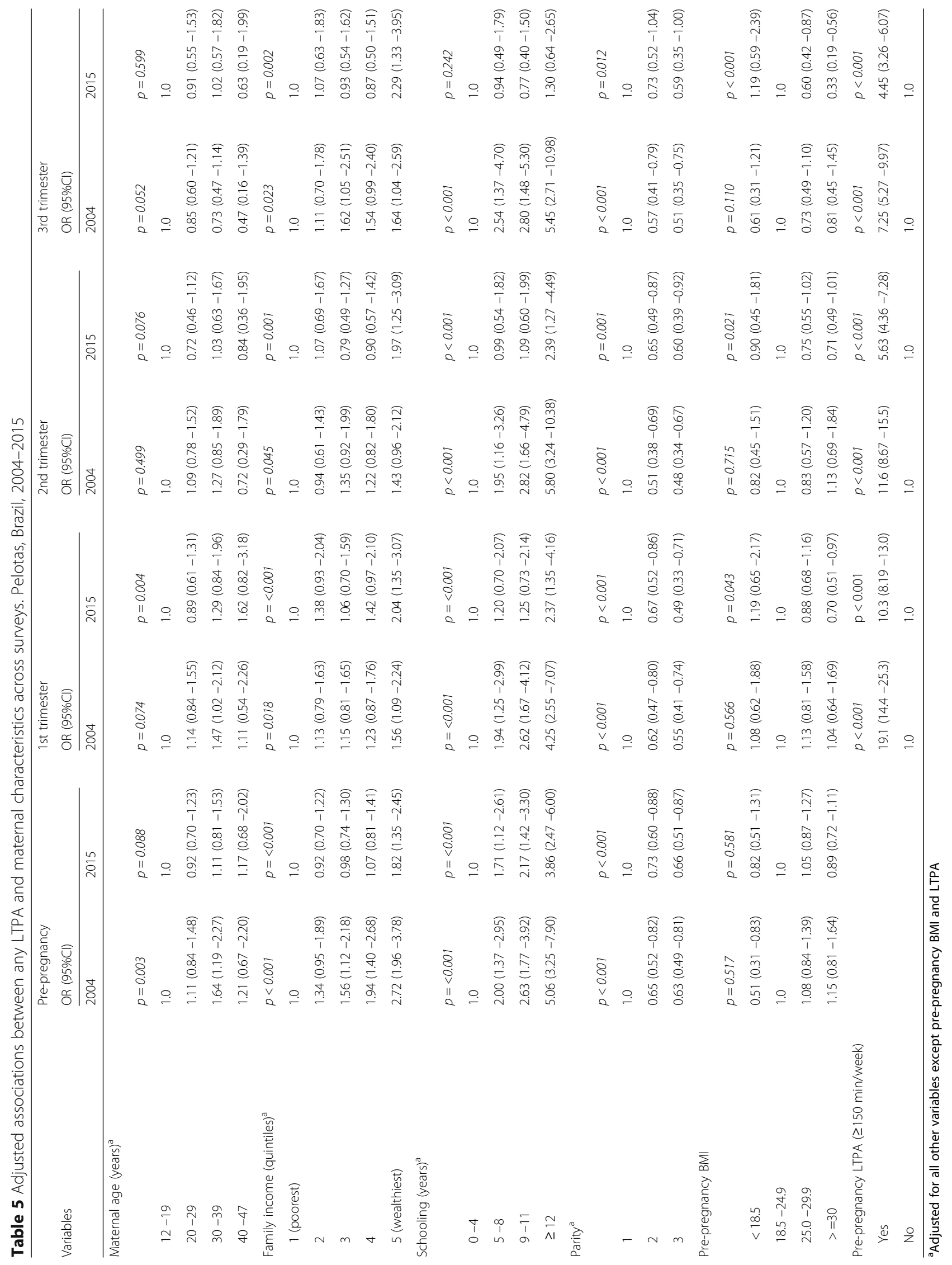




\section{Discussion}

The present study compared LTPA levels prior to and during pregnancy among Brazilian women over an 11year period (2004-2015). The findings indicated an increase in the proportion of women engaged in prepregnancy LTPA over the period. On the other hand, no change in LTPA levels was observed for the first and second trimesters of pregnancy while a decline was observed for the last trimester from 2004 to 2015. Overall, major decreases in the prevalence of LTPA were observed among young mothers (20 to 29 years), classified in the third and fourth quintiles of family income, with higher schooling ( $\geq 9$ years of formal education), firsttime mothers, obese according to their pre-pregnancy BMI and who were engaged in recommended levels of LTPA before pregnancy. Although the total prevalence of LTPA remained unchanged in 2015 for the first and second trimesters of pregnancy as compared to 2004, declines in LTPA patterns were observed for different subgroups of the population, which followed very similar patterns observed for the third trimester of pregnancy.

The increase in the number of women engaged in prepregnancy LTPA observed in the most recent cohort study is in line with LTPA trend patterns described for Brazilian adults based on recent data from a national surveillance system [20]. From 2006 to 2012, the prevalence of recommended LTPA increased from $12.8 \%$ to $14.9 \%$ among the adult Brazilian population. During this period, a marked increase was observed particularly among young adults, which seems to encompass the population of women of childbearing age who are part of our cohort. On the other hand, our findings showed that pre-pregnancy LTPA has become a less important predictor of pregnancy LTPA over the period, with a greater number of previously active women discontinuing or decreasing LTPA engagement during pregnancy in 2015, as compared with 2004.

National efforts to promote physical activity were recently intensified in the country in an attempt to decrease the burden of non-communicable diseases and this may partly explain the increase in pre-pregnancy LTPA levels observed [21]. Nevertheless, as we can observe through our findings, the possible increased population awareness about LTPA benefits does not seem to be translated into practice when it comes to the pregnancy period. This is particularly relevant because inactivity during pregnancy is known to be associated with increased risks of maternal complications [5], also influencing maternal long-term chronic disease risk and susceptibility in the offspring [10, 22]. Therefore, as inactivity during pregnancy tends to remain in the postpartum period and beyond [3, 4], the implementation of physical activity interventions that target pregnant women may positively influence future overall physical activity trends and might be considered by policy makers as part of a strategy to effectively reduce the burden of non-communicable diseases at the population level.

Data from the USA for 1999-2006 on national physical activity trends among pregnant women had shown that participation in any moderate LTPA increased about $24 \%$ over the 7-year period (from $46.8 \%$ in 1999 to $58.0 \%$ in 2006) [16]. At the same time, the proportion of women meeting recommendations for LTPA remained stable [16]. Whilst the reference period assessed by the authors does not allow for a direct comparison with our findings, the increasing trend in any LTPA patterns reported among USA pregnant women goes in the opposite direction of a decrease in both any and recommended levels of LTPA during pregnancy found in the present study. Besides, the proportion of women engaged in LTPA reported by the authors was much higher than estimates found in the current study among Brazilian pregnant women.

To compare our data, however, we need to consider that research on physical activity during pregnancy and its influence on maternal-child health outcomes started at least three decades ago in high-income countries and LTPA promotion among pregnant women seems to have been an issue of concern for some time [23]. While the American College of Obstetricians and Gynecologists guidelines for physical activity during pregnancy were initially released in 1985 and have been discussed and reaffirmed over time, in Brazil, recommendations to provide guidance to health care providers are still nonexistent; the available scientific knowledge regarding physical activity during pregnancy seems not being translated to clinical practice and adopted by the population.

A similar pattern of disparity in physical activity promotion progress can be described if we take a look at trends in LTPA participation among the adult population worldwide. While increases in LTPA patterns have been reported for adults living in high-income countries in the past 20-30 years [24], in Brazil they recently began to be observed. In this sense, it is possible that advances made in LTPA patterns among the Brazilian adult population are too recent to have also impacted LTPA patterns during pregnancy given that interventions to target specifically pregnant women are still missing in the country. Moreover, LTPA participation during pregnancy is a more complex behavior to modify once it is often surrounded by misconceptions and uncertainty regarding its benefits to the mother-child health as well as the symptoms and limitations of the gestational period [25].

Among the barriers preventing women to engage in LTPA practice during pregnancy, the lack of knowledge to make decisions about exercise is one of the most reported in the literature [25]. In this context, being the most 
influent source of information for pregnant women, healthcare providers play an important role in providing pregnant women with the necessary knowledge and support to engage in antenatal physical activity [26]. However, even in high income countries where LTPA during pregnancy is more frequently promoted, results from a study had shown that over a third of health professionals were not confident in their knowledge regarding benefits and risks of LTPA during pregnancy [27]. Importantly, in the present study counseling made by prenatal healthcare professionals on LTPA practice declined over time. Therefore, there is an urgent need to increase awareness of physical activity benefits during pregnancy and the available recommendations among healthcare providers. In this context, implementation and dissemination research is and essential framework to further understand the gap between knowledge and practice [28] related to LTPA promotion during pregnancy [29].

Intriguingly, in our study a pattern of decline in pregnancy LTPA levels over the period was particularly observed for those mothers belonging to the groups of high income and education. Since healthy behaviors tend to be first adopted by those who have greater access to information, education and economic resources for prevention, an opposite trend would be expected compared to what we observed [30]. Smoking patterns among pregnant women in Brazil, for example, has been declining markedly since the 80's, with greater and faster declines being observed among women with higher income [31]. However, tobacco-control policies are more advanced in the country compared to physical activity promotion, and the increased awareness about the harmful effects of smoking during pregnancy seems to have been translated into a favorable change of behavior by the population. In the present study smoking during pregnancy decreased $37 \%$ in this 11 -year period.

To better understand the changes observed in the present study we are unable to dissociate the marked changes in socioeconomic, demographic and health characteristics during the time period assessed. The observed improvement in income and schooling was accompanied by an increase in maternal obesity and gestational diabetes that is certainly playing a role in LTPA changes observed and may also suggest a population undergoing a nutrition transition. The lower LTPA during pregnancy among pre-pregnancy obese women in 2015, especially in the last gestational trimester, are in agreement with a recent study showing decreased levels of exercise as pregnancy advances, particularly among pre-pregnancy obese women [32]. Besides, maternal obesity itself is associated with a higher risk of clinical complications such as gestational diabetes, hypertension and preeclampsia [33-35]. Accordingly, in the 2015 cohort study the prevalence of gestational diabetes was
$17 \%$ among pre-pregnancy obese women compared to $4.1 \%$ among women with normal BMI, while the prevalence of hypertension was $45.9 \%$ and $16.8 \%$, respectively (data not shown).

From 2004 to 2015, a significant decrease in LTPA patterns was also observed among obese women and this was especially evident in the last trimester of pregnancy, which might be related to an increase in the severity of maternal complications over time. In this sense, it is also possible that the increase trend in obesity and its related health complications had imposed challenges in the prenatal care assistance, having a negative impact in the confidence to counseling LTPA among healthcare providers. Although LTPA participation during pregnancy had been shown to have benefits in the prevention and/or management of gestational diabetes mellitus, excessive gestational weight gain and hypertensive disorders [5, 36-38], a clear disconnection between scientific evidence and clinical practice exists and the lack of knowledge of risks and benefits of LTPA during pregnancy is very common [29]. Furthermore, prenatal healthcare providers perceived barriers have been shown to increase when providing antenatal counseling for obese women [39].

Regarding correlates of LTPA during pregnancy, overall our findings support previous studies reporting positive associations between LTPA with maternal education, income and pre-pregnancy LTPA as well as a negative association with parity in both time points [40, 41]. However, suitable changes in the shape of associations could be observed over the period. While in 2004 the associations between LTPA and schooling was clearly linear, in 2015 only women from the highest schooling category had a greater probability of being active compared to women in the reference group (0-4 years of formal education). Moreover, although pre-pregnancy LTPA was the strongest predictor of LTPA engagement during pregnancy in both cohorts, the strength of the association decreased from 2004 to 2015.

Similar to other studies reporting on type of LTPA among pregnant women [42, 43], walking was the most prevalent LTPA during pregnancy in both cohorts. However, a significant decrease in the proportion of pregnant women engaged in walking was observed from 2004 to 2015 while other activities such as weight training, water gymnastics, aerobics and dancing increased. This shift in the type of LTPA practiced during pregnancy might reflect important changes in women's beliefs and preferences over time. It might be possible that pregnant women engaged in LTPA nowadays are enough confident about its benefits and, therefore, feeling comfortable to explore a broader range of activities that used to be avoided in the past due to safety concerns and lack of knowledge. Changes in the type of LTPA performed over time with an 
increase in gym-related activities have been reported in monitoring studies conducted with other populations and may also be reflecting a generational effect [44].

\section{Strengths and limitations}

The similarity of data collection methods between both surveys combined with the high response rates, are certainly the major strengths of the present study. The use of the same inclusion criteria and methodologies over an 11-year period provides reliable findings. Besides, LTPA patterns were measured during the different trimesters of pregnancy allowing the distinction of specific changes that happened across gestational trimesters.

Some limitations of the study need to be taken into consideration while interpreting the findings. First, instruments based on self-report such as the one used in this study, might overestimate physical activity levels. However, as this limitation was equally present in both surveys, comparability over time was not impaired. Second, the intensity of the reported leisure-time physical activities was not measured and for this reason we could not assess its changes over time. Yet, the lack of information on intensity was purposeful given that intensity prompts (amount of moderate or vigorous-intensity activities) would not be ideal in a retrospective analysis-women might remember how much they practiced some months ago, but probably not the intensity of the activities performed. Third, the retrospective assessment of LTPA could result in recall bias. But in the worst scenario mothers had to report their LTPA patterns 9 months before the interview (pre-pregnancy LTPA), which is considered a reasonable period since long-term maternal recall of pregnancy-related events has been suggested to be highly accurate [45]. Lastly, it should also be noted that the prevalence estimates reported in the present study might have being influenced by the fact that we excluded 141 mothers from the 2015 Birth Cohort Study who took part in an exercise intervention during pregnancy and who were not reaching recommended levels of LTPA during recruitment (inclusion criteria in the trial). Nevertheless, given the low probability of previously inactive women starting to practice physical activity during the pregnancy period [46] we expect a slight overestimation in the observed prevalence of LTPA during pregnancy in 2015. Consequently, the decline trends observed could be worse if including this sample of women.

\section{Conclusions and recommendations}

The present study contributed to fill the knowledge gap on population LTPA trends during pregnancy. Our findings showed a significant increase in the proportion of women engaged in LTPA prior to pregnancy. On the other hand, no improvements in LTPA levels during pregnancy were observed over the 11-year period assessed, whereas a significantly decrease was observed for the third trimester of pregnancy. Future research might still include assessment of mother-child health benefits of physical activity during pregnancy, but must also address challenges in terms of implementation and dissemination of physical activity promotion. Moving towards to action, there is pressing need for intervention strategies aimed at increasing LTPA levels among pregnant women to reverse this trend and help stall the progression of its negative health consequences. Women should be encouraged to view the preconception period and pregnancy as opportunities to adopt healthy behaviors such as LTPA that could be maintained throughout life. In this context, healthcare providers involved in prenatal care play an essential role in advising women on LTPA benefits during pregnancy and encouraging them to start or continue exercising.

\section{Abbreviations \\ LTPA: Leisure-time physical activity; BMI: Body Mass Index}

\section{Acknowledgements}

The authors want to thank all funding agencies, the mothers who consented to be part of both birth cohort studies and the research team who collected data and supervised the study.

\section{Funding \\ The 2004 Pelotas Birth Cohort Study was financed by the Division of Child and Adolescent Health and Development of the World Health Organization (WHO, Geneva), by the Conselho Nacional de Desenvolvimento Científico e Tecnológico (CNPq, Brazil), and by the Pastoral da Criança (Catholic NGO, Curitiba, Brazil). The Wellcome Trust and Associação Brasileira de Saúde Coletiva (ABRASCO) have supported the analysis of both cohort studies.}

\section{Availability of data and material}

The data that support the findings of this study are available from Postgraduate Program in Epidemiology, Federal University of Pelotas, Brazil, but restrictions apply to the availability of these data, which were used under license for the current study, and so are not publicly available. Data are however available from the authors upon reasonable request and with permission of the Postgraduate Program in Epidemiology, Federal

University of Pelotas, Brazil.

\section{Authors 'contributions}

CVNC wrote the paper and conducted statistical data analysis. AM, IS, AB and MRD supervised the fieldwork of the 2004 Birth Cohort Study while CVNC, MRD, ICS, DG and ADB supervised the fieldwork of the 2015 Birth Cohort study. All authors critically revised the paper and approved the final versions of the manuscript to be published.

\section{Competing interests}

The authors declare that they have no competing interests.

\section{Consent for publication}

Not applicable.

\section{Ethics approval and consent to participate}

The Medical Research Ethics Committee and the Superior School of Physical Education Committee of the Federal University of Pelotas approved the studies protocols under the numbers 4.06.01.116 and 522.064 for the 2004 and 2015 cohorts, respectively. The interviews were conducted only after informing mothers of the study objectives and a signed informed consent was obtained. 


\section{Author details}

${ }^{1}$ Postgraduate Program in Epidemiology, Federal University of Pelotas, Pelotas, Brazil. ${ }^{2}$ Postgraduate Program in Physical Education, Federal University of Pelotas, Pelotas, Brazil. ${ }^{3}$ International Center for Equity in Health, Federal University of Pelotas, Pelotas, Brazil. ${ }^{4}$ Centre for Global Child Health, The Hospital for Sick Children, Toronto, Canada. ${ }^{5}$ Department of Paediatrics and Dalla Lana School of Public Health, University of Toronto, Toronto, Canada. ${ }^{6}$ Department of Preventive Medicine, Faculty of Medicine, University of São Paulo, São Paulo, Brazil.

\section{Received: 19 October 2016 Accepted: 13 January 2017} Published online: 25 January 2017

\section{References}

1. Ding D, Lawson KD, Kolbe-Alexander TL, Finkelstein EA, Katzmarzyk PT, van Mechelen W, Pratt M. The economic burden of physical inactivity: a global analysis of major non-communicable diseases. Lancet (London, England). 2016; 388(10051):1311-24.

2. Sallis JF, Bull F, Guthold R, Heath GW, Inoue S, Kelly P, Oyeyemi AL, Perez LG, Richards J, Hallal PC. Progress in physical activity over the Olympic quadrennium. Lancet (London, England). 2016;388(10051):1325-36.

3. Engberg E, Alen M, Kukkonen-Harjula K, Peltonen JE, Tikkanen HO, Pekkarinen $\mathrm{H}$. Life events and change in leisure time physical activity: a systematic review. Sports medicine (Auckland, NZ). 2012;42(5):433-47.

4. Coll C, Domingues M, Santos I, Matijasevich A, Horta BL, Hallal PC. Changes in Leisure-Time Physical Activity From the Pre-Pregnancy to the Postpartum Period: 2004 Pelotas (Brazil) Birth Cohort Study. Journal of physical activity \& health. 2015;12(4):361-65.

5. da Silva SG, Ricardo LI, Evenson KR, Hallal PC. Leisure-Time Physical Activity in Pregnancy and Maternal-Child Health: A Systematic Review and MetaAnalysis of Randomized Controlled Trials and Cohort Studies. Sports medicine (Auckland, NZ) 2016. [Epub ahead of print].

6. Wolf HT, Owe KM, Juhl M, Hegaard HK. Leisure time physical activity and the risk of pre-eclampsia: a systematic review. Matern Child Health J. 2014; 18(4):899-910.

7. Teychenne M, York R. Physical activity, sedentary behavior, and postnatal depressive symptoms: a review. Am J Prev Med. 2013;45(2):217-27.

8. Harrod CS, Chasan-Taber L, Reynolds RM, Fingerlin TE, Glueck DH, Brinton JT, Dabelea D. Physical activity in pregnancy and neonatal body composition: the Healthy Start study. Obstet Gynecol. 2014;124(2 Pt 1):257-64.

9. Pivarnik JM, Chambliss HO, Clapp JF, Dugan SA, Hatch MC, Lovelady CA, Motolla MF, Williams MA. Impact of physical activity during pregnancy and postpartum on chronic disease risk. Medicine and science in sports and exercise. 2006;38(5):989-1006.

10. Blaize AN, Pearson KJ, Newcomer SC. Impact of Maternal Exercise during Pregnancy on Offspring Chronic Disease Susceptibility. Exerc Sport Sci Rev. 2015;43(4):198-203.

11. Evenson KR, Barakat R, Brown WJ, Dargent-Molina P, Haruna M, Mikkelsen EM, Mottola MF, Owe KM, Rousham EK, Yeo S. Guidelines for Physical Activity during Pregnancy: Comparisons From Around the World. American Journal Of Lifestyle Medicine. 2014;8(2):102-21.

12. Committee Opinion No ACOG. 650: Physical Activity and Exercise During Pregnancy and the Postpartum Period. Obstet Gynecol. 2015;126(6):e135-142.

13. Domingues MR, Barros AJ. Leisure-time physical activity during pregnancy in the 2004 Pelotas Birth Cohort Study. Rev Saude Publica. 2007:41(2):173-80.

14. Padmapriya N, Shen L, Soh SE, Shen Z, Kwek K, Godfrey KM, Gluckman PD, Chong YS, Saw SM, Muller-Riemenschneider F. Physical Activity and Sedentary Behavior Patterns Before and During Pregnancy in a Multi-ethnic Sample of Asian Women in Singapore. Matern Child Health J. 2015;19(11):2523-35.

15. Di Fabio DR, Blomme CK, Smith KM, Welk GJ, Campbell CG. Adherence to physical activity guidelines in mid-pregnancy does not reduce sedentary time: an observational study. Int J Behav Nutr Phys Act. 2015;12:27.

16. Evenson KR, Wen F. National trends in self-reported physical activity and sedentary behaviors among pregnant women: NHANES 1999-2006. Prev Med. 2010;50(3):123-8

17. Mudd LM, Owe KM, Mottola MF, Pivarnik JM. Health benefits of physical activity during pregnancy: an international perspective. Med Sci Sports Exerc. 2013;45(2):268-77.

18. Santos IS, Barros AJ, Matijasevich A, Domingues MR, Barros FC, Victora CG. Cohort profile: the 2004 Pelotas (Brazil) birth cohort study. Int J Epidemiol. 2011;40(6):1461-8
19. Domingues MR, Bassani DG, da Silva SG, Coll Cde V, da Silva BG, Hallal PC. Physical activity during pregnancy and maternal-child health (PAMELA): study protocol for a randomized controlled trial. Trials. 2015;16:227.

20. Mielke Gl, Hallal PC, Malta DC, Lee IM. Time trends of physical activity and television viewing time in Brazil: 2006-2012. Int J Behav Nutr Phys Act. 2014;11:101.

21. Malta DC, Morais Neto OL, Silva Junior JB. Apresentação do plano de ações estratégicas para o enfrentamento das doenças crônicas não transmissíveis no Brasil, 2011 a 2022. Epidemiologia e Serviços de Saúde. 2011;20:425-38.

22. Bao W, Tobias DK, Bowers K, Chavarro J, Vaag A, Grunnet LG, Strom M, Mills J, Liu A, Kiely M, et al. Physical activity and sedentary behaviors associated with risk of progression from gestational diabetes mellitus to type 2 diabetes mellitus: a prospective cohort study. JAMA Intern Med. 2014;174(7):1047-55.

23. Downs DS, Chasan-Taber L, Evenson KR, Leiferman J, Yeo S. Physical Activity and Pregnancy: Past and Present Evidence and Future Recommendations. Res Q Exerc Sport. 2012;83(4):485-502.

24. Hallal PC, Andersen LB, Bull FC, Guthold R, Haskell W, Ekelund U. Global physical activity levels: surveillance progress, pitfalls, and prospects. Lancet (London, England). 2012;380(9838):247-57.

25. Coll CV, Domingues MR, Goncalves H, Bertoldi AD: Perceived barriers to leisure-time physical activity during pregnancy: A literature review of quantitative and qualitative evidence. Journal of science and medicine in sport / Sports Medicine Australia. 2016;20(1):17-25.

26. Yamamoto A, McCormick MC, Burris HH. US provider-reported diet and physical activity counseling to pregnant and non-pregnant women of childbearing age during preventive care visits. Matern Child Health J. 2014;18(7):1610-8.

27. Leiferman J, Gutilla M, Paulson J, Pivarnik J. Antenatal physical activity counseling among healthcare providers. Open Journal of Obstetrics and Gynecology. 2012;2:9.

28. Glasgow RE, Vinson C, Chambers D, Khoury MJ, Kaplan RM, Hunter C. National Institutes of Health approaches to dissemination and implementation science: current and future directions. Am J Public Health. 2012;102(7):1274-81.

29. Watson ED, Oddie B, Constantinou D. Exercise during pregnancy: knowledge and beliefs of medical practitioners in South Africa: a survey study. BMC Pregnancy Childbirth. 2015;15:245

30. Victora CG, Vaughan JP, Barros FC, Silva AC, Tomasi E. Explaining trends in inequities: evidence from Brazilian child health studies. Lancet (London, England). 2000;356(9235):1093-8.

31. Silveira MF, Matijasevich A, Menezes AM, Horta BL, Santos IS, Barros AJ, Barros FC, Victora CG. Secular trends in smoking during pregnancy according to income and ethnic group: four population-based perinatal surveys in a Brazilian city. BMJ Open. 2016;6(2):e010127.

32. Daly N, Mitchell C, Farren M, Kennelly MM, Hussey J, Turner MJ. Maternal obesity and physical activity and exercise levels as pregnancy advances: an observational study. Irish Journal of Medical Science (1971 -). 2016;185(2):357-70.

33. Torloni MR, Betran AP, Horta BL, Nakamura MU, Atallah AN, Moron AF, Valente O. Prepregnancy BMI and the risk of gestational diabetes: a systematic review of the literature with meta-analysis. Obesity Reviews. 2009;10(2):194-203.

34. Spradley FT, Palei AC, Granger JP. Increased risk for the development of preeclampsia in obese pregnancies: weighing in on the mechanisms.

Am J Physiol Regul Integr Comp Physiol. 2015;309(11):R1326-1343.

35. Rahman MM, Abe SK, Kanda M, Narita S, Rahman MS, Bilano V, Ota E, Gilmour S, Shibuya K. Maternal body mass index and risk of birth and maternal health outcomes in low- and middle-income countries: a systematic review and meta-analysis. Obesity Reviews. 2015;16(9):758-70.

36. Padayachee C, Coombes JS. Exercise guidelines for gestational diabetes mellitus. World J Diabetes. 2015;6(8):1033-44.

37. Muktabhant B, Lawrie TA, Lumbiganon P, Laopaiboon M. Diet or exercise, or both, for preventing excessive weight gain in pregnancy. Cochrane Database Syst Rev. 2015;6:Cd007145.

38. Chawla S, Anim-Nyame N. Advice on exercise for pregnant women with hypertensive disorders of pregnancy. Int J Gynaecol Obstet. 2015;128(3):275-9.

39. Kominiarek MA, Gay F, Peacock N. Obesity in Pregnancy: A Qualitative Approach to Inform an Intervention for Patients and Providers. Matern Child Health J. 2015;19(8):1698-712.

40. Nascimento SL, Surita FG, Godoy AC, Kasawara KT, Morais SS. Physical Activity Patterns and Factors Related to Exercise during Pregnancy: A Cross Sectional Study. PLoS One. 2015;10(6):e0128953.

41. Gaston A, Cramp A. Exercise during pregnancy: a review of patterns and determinants. J Sci Med Sport. 2011;14(4):299-305.

42. Evenson KR, Savitz DA, Huston SL. Leisure-time physical activity among pregnant women in the US. Paediatr Perinat Epidemiol. 2004;18(6):400-7. 
43. Santos PC, Abreu S, Moreira C, Santos R, Ferreira M, Alves O, Moreira P, Mota J. Physical Activity Patterns During Pregnancy in a Sample of Portuguese Women: A Longitudinal Prospective Study. Iranian Red Crescent Medical Journal. 2016:18(3):e22455.

44. Coll Cde V, Knuth AG, Bastos JP, Hallal PC, Bertoldi AD. Time trends of physical activity among Brazilian adolescents over a 7-year period. J Adolesc Health. 2014;54(2):209-13.

45. Tomeo CA, Rich-Edwards JW, Michels KB, Berkey CS, Hunter DJ, Frazier AL, Willett WC, Buka SL. Reproducibility and validity of maternal recall of pregnancy-related events. Epidimiology. 1999;10(6):774-7.

46. Huberty JL, Buman MP, Leiferman JA, Bushar J, Adams MA. Trajectories of objectively-measured physical activity and sedentary time over the course of pregnancy in women self-identified as inactive. Preventive Medicine Reports. 2016;3:353-60.

Submit your next manuscript to BioMed Central and we will help you at every step:

- We accept pre-submission inquiries

- Our selector tool helps you to find the most relevant journal

- We provide round the clock customer support

- Convenient online submission

- Thorough peer review

- Inclusion in PubMed and all major indexing services

- Maximum visibility for your research

Submit your manuscript at www.biomedcentral.com/submit
Biomed Central 